\title{
The effects of immersive garden experience on the health care to elderly residents with mild-to-moderate cognitive impairment living in nursing homes after the COVID-19 pandemic
}

\author{
Chung-Heng Hsieh ${ }^{1} \cdot$ Cheng-Min Chen ${ }^{2}$ Ju-Yuan Yang ${ }^{1} \cdot$ Yen-Ju Lin ${ }^{3} \cdot$ Man-Li Liao ${ }^{4} \cdot$ Ke-Hsin Chueh ${ }^{5,6}$
}

Received: 26 June 2021 / Revised: 12 September 2021 / Accepted: 16 September 2021 / Published online: 6 October 2021

(c) International Consortium of Landscape and Ecological Engineering 2021

\begin{abstract}
Elderly patients living in long-term care facilities have been restricted from leaving to comply with social distancing guidelines during the COVID-19 pandemic. This has led to a worsening of disorders, such as anxiety and depression. This study aims to understand the health benefits of an immersive garden experience to elderly nursing home residents with mild-tomoderate cognitive impairments. Virtual reality devices were used to provide immersive garden experiences for the residents who were unable to go outside. The heart rate and heart rate variability (HRV) data of the participants of the participants were collected using biofeedback instruments, and changes in the low frequency/high frequency (LF/HF) and the standard deviation of the NN interval (SDNN) values caused by immersive garden experiences were discussed. The results show that the immersive garden experiences were beneficial to these elderly residents. Within $6 \mathrm{~min}$ of completing the experiment, we found that the heart rates of participants had dropped slightly, while SDNN and HF values continued to rise. SDNN values before and after the experiment demonstrated a statistically significant improvement. Furthermore, participants expressed their satisfaction with the video intervention program. The results indicated that nursing homes can provide immersive landscape experiences to help increase HRV and SDNN of their elderly residents. This will not only help these residents recall beautiful memories of their past, but will also improve their quality of life.
\end{abstract}

Keywords 360 -Degree garden video $\cdot$ Dementia $\cdot$ Heart rate variability $\cdot \mathrm{LF} / \mathrm{HF} \cdot \mathrm{SDNN}$

Ke-Hsin Chueh

125598@mail.fju.edu.tw

Chung-Heng Hsieh

wn1172@hotmail.com

Cheng-Min Chen

hihiisbel0604@gmail.com

Ju-Yuan Yang

rona8639@gmail.com

Yen-Ju Lin

llinyanru@gmail.com

Man-Li Liao

mandy130623@gmail.com

1 Department of Landscape Architecture, College of Art, Fu Jen Catholic University, No. 510, Zhongzheng Rd., Xinzhuang Dist., New Taipei City 24205, Taiwan
2 Bachelor Degree Program of Art and Culture Creation, Fu Jen Catholic University, No. 510, Zhongzheng Rd., Xinzhuang Dist., New Taipei City 24205, Taiwan

3 Department of Landscape Architecture, College of Art, Fu Jen Catholic University, No. 510, Zhongzheng Rd., Xinzhuang Dist., New Taipei City 24205, Taiwan

4 Fu Jen Catholic University Hospital, Fu Jen Catholic University, No. 69, Guizi Rd., Taishan, New Taipei City, Taiwan

5 Graduate Institute of Landscape Architecture \& Recreation Management, National Pingtung University of Science and Technology, Pingtung, Taiwan

6 Department of Nursing, College of Medicine, Fu Jen Catholic University, No. 510, Zhongzheng Rd., Xinzhuang Dist., New Taipei City 24205, Taiwan 


\section{Introduction}

Owing to medical technology advances, the proportion of elderly people has rapidly increased in recent years. The elderly population usually suffers from a gradual decline in psychological functions, coupled with the fact that their children are often too busy with work. As a result, the number of long-term care (LTC) institutions has been increasing consistently. In the United States in 2016, there were 65,600 LTC (defined as stays of longer than 100 days) providers taking care of 1,605,500 residents (Harris-Kojetin et al. 2019). The ratio of institutional nursing beds to the elderly population in China was 1:39 in 2014 (Economic and Social Commission for Asia and the Pacific 2016). In Taiwan, there are presently 1091 LTC institutions that take care of 50,966 residents.

Past studies have indicated that depression affects almost $30 \%$ of LTC residents and $23.3 \%$ of them suffer from depression along with cognitive impairment (Hoben et al. 2019). Lee et al. (2002) emphasized that the environments of nursing homes must be strictly controlled, meaning that nursing homes residents are unable to enter and leave freely. The prevalence of severe loneliness reported by care home residents is more than twice that of residents at home (Victor 2012). Lee et al. (2013) argued that elder people moving into residential care often experience stress and anxiety due to not feeling confident in their decision to move, living in constant fear of losing their memory, and limited expectations for their future. This gradually leads to depression, boredom, and loneliness. LTC residents are three to four times more likely to suffer from depression and other related disorders compared to elderly people living in residential neighborhoods (Jongenelis et al. 2004). Huang et al. (2014) stated that the prevalence of depression in the elderly population of Taiwan living in nursing homes ranged from 39 to $82 \%$, far surpassing that of Western countries (8.1-24\%). Man et al. (2012) mentioned that about $10-12 \%$ of elderly people over 65 years of age living in Taiwan suffered from at least one mental disorder, such as depression. This demonstrates that the psychological health of the elderly in nursing homes has become an important issue.

Depression might lead to an autonomic nervous system (ANS) disorder, affect heart rate variability (HRV), and increase the risk of cardiovascular diseases. Kop et al. (2010) noted that an abnormal HRV can accelerate arrhythmia, cause arteriosclerosis, and increase the possibility of cardiovascular disease. HRV measurement is a non-invasive method used to evaluate ANS. Its clinical applications and changing standards can verify the health statuses of patients. A low or falling HRV means that the ANS is functioning abnormally, suggesting failing health (Catai et al. 2020). The standard deviation of the NN interval (SDNN) is the most representative parameter of HRV. A higher SDNN means a higher HRV and stronger parasympathetic nerve activity. On the contrary, a lower SDNN means a lower HRV and stronger sympathetic nerve activity. Kurita et al. (2013) previously conducted an HRV analysis on elderly residents living in LTC institutions and discovered that when SNDD exceeded 65, the residents tended to live longer. Kleiger et al. (1987) analyzed patients with myocardial infarctions for $24 \mathrm{~h}$ and found that SDNN could be used to predict acute myocardial infarctions; they also found that the death rate of patients with low SDNN was 5.3 times higher than that of patients with high SDNN. La Rovere et al. (1998) also found that a low heart rate signified a higher rate of cardiovascular disease. Bilchick et al. (2002) mentioned that patients with SDNN $<65.3 \mathrm{~ms}$ had a higher sudden death rate. Therefore, if it is possible to increase the SDNN of patients, this can greatly benefit their physiological health. The frequency parameter, specifically low frequency/high frequency (LF/HF), is the index used for measuring sympathetic and parasympathetic nerve activity, respectively. The sympathetic nervous system (SNS) is responsible for increasing heart rate and shortness of breath, slowing down digestion, and increasing muscle tension, putting people into a state of anxiousness and tension. On the other hand, the parasympathetic nervous system (PNS) slows heart rate and breathing, promotes digestion, and reduces muscle tension, allowing people to relax. All in all, it would be favorable to improve ANS activity through either raising SDNN values or maintaining standard LF/ HF values. Doing so will help reduce anxiety in patients and reduce the risk of cardiovascular disease.

The care needs of elders with chronic conditions have increased the medical burden in many developed countries. The Health Promotion Administration (2018) highlighted that the medical expenditure for treating elderly patients with chronic diseases has increased each year in Taiwan. It is estimated that nearly $70 \%$ of the elderly population suffers from two chronic diseases and $50 \%$ of the elderly population suffers from three chronic diseases, the majority of which are cardiovascular diseases (e.g., hypertension) and diabetes. Chiu et al. (2019) stated that the proportion of medical resources consumed by the elderly over 65 years of age in Taiwan has increased from 28.8 to $37.3 \%$ and medical expenditure has increased by nearly $30 \%$. According to the report of the Health Promotion Administration (2017), up to 27,550 elderly patients over the age of 65 years in Taiwan received treatment for cardiovascular diseases, the highest number among all age groups, accounting for a high proportion of modern medical expenditures. As such, an opportunity to increase ANS activity and maintain cardiovascular health of elderly 
residents would allow the government to lower the healthcare budget for chronic diseases.

Relative studies (Browning et al. 2020; Craske et al. 2019; Jerdan et al. 2018; Maples-Keller et al. 2017; Palanica et al. 2019; White et al. 2018) have shown that providing nature experiences could provide health benefits. Lin et al. (2018) believed that city gardens could engender biophilia for their participants by increasing exposure, positive interactions, and knowledge of nature. Not only does this have a positive effect on their emotions, but it also strengthens their bodies and has positive psychological benefits (Schermer 2014). Given that there is a limitation on living space, recent studies have begun to emphasize that experiencing immersive virtual reality (VR) will improve an individual's health. Valtchanov and Ellard (2015) compared the effects of a virtual versus artificial nature environment in their studies, finding that a virtual environment was able to enhance the creativity of their participants. Kim et al. (2017) indicated that VR could increase positive emotional reactions, regardless of whether the subjects were healthy or suffered from Parkinson's disease. Liszio et al. (2018) found that the psychological benefits brought about by virtual reality were better than those of watching regular videos. Mattila et al. (2020) also highlighted that high-quality nature VR has a significant effect on emotional and attention recovery.

In the field of clinical trials, Palanica et al. (2019) indicated that watching stimulating videos (nature VR videos) had a significant effect on the creative thinking of the participants. For specific subjects, Craske et al. (2019) divided clinical trials for anxiety disorders into positive affect treatment (PAT) and negative affect treatment (NAT), finding that VR could provide patients with a positive experience and could be used to help patients meditate to treat their depression while increasing their well-being. Manera et al. (2016) found that when put through a virtual nature experience, dementia patients expressed higher satisfaction, lower anxiety, and less discomfort. Man et al. (2012) found that dementia patients were able to improve their memory function through a VR intervention program. Doniger et al. (2018) found that VR could improve the cognitive ability of patients with Alzheimer's disease.

Although many studies have recently begun exploring the benefits of VR nature experiences in patients, most studies in dementia patients in LTC facilities have elected to evaluate the benefits of immersive experiences through interviews or questionnaires. However, for elderly people with dementia who have cognitive impairment issues, it is relatively difficult to fill out questionnaires. Therefore, it is still open to discussion whether questionnaires filled out by these subjects are true representations of their psychological states. As such, it is necessary to evaluate the benefits of VR through other more objective measures.
Currently, nursing homes must consider the safety of their residents due to COVID-19; therefore, elderly residents are now generally unable to go outside. This has led to more elderly residents becoming depressed and refusing to cooperate with health-care employees. Past studies have shown that virtual nature has positive psychological benefits (Liszio et al. 2018; Valtchanov and Ellard 2015). Mattila et al. (2020) revealed that allowing subjects to experience a familiar virtual forest environment had a restorative effect on their health. This is important for people who do not have easy access to forests or other natural environments. Furthermore, Kemperman and Timmermans (2014) indicated that the elderly prefer landscapes such as flower or vegetable gardens similar to their daily lives. Thus, whether the immersive garden experiences that the elderly residents like to stay in are equally beneficial to the individuals in nursing homes needs to be further explored.

This study carried out an experiment with the elderly residents of nursing homes as the subjects, allowing them to partake in immersive experience technology that uses garden landscapes as the medium. This study measures the differences between SDNN and LF/HF before, during, and after the 360-degree video viewing and records the feelings and opinions of the subjects after the experiment procedure. The experiment was designed to explore the physiological and psychological benefits of immersive garden experiences.

\section{Materials and methods}

\section{Subject of study}

This study used a convenience sampling method. The researchers communicated with two nursing homes in New Taipei City before the experiment. This study was then approved by the Institutional Review Board of Fu Jen Catholic University (IRB: C107072). All the residents of the nursing homes who participated in the present study were older than 50 years of age. Residents who were taking antihypertensive drugs and who were currently receiving psychiatric treatment for emotional disorders were excluded. The researcher explained the experimental procedure to potential participants and only after the participants had signed the consent form could the study be carried out. Researchers with professional nursing training used the Mini Mental State Examination (MMSE), which was developed by Folstein et al. (1975) to screen test subjects. The present study also referred to Lin et al. (2007) and set the maximum score at 30 points: 25 to 30 points was defined as normal, 21 to 24 points as mild dementia, 14 to 20 points as moderate dementia, and under 12 points as severe dementia. A total of 21 subjects who scored more than 13 points but less than 24 were selected, that is, those with mild-to-moderate 
dementia-were selected; this comprised the final list of participants for the video intervention program. In the end, 14 subjects completed the experimental procedure with the researchers. Four subjects withdrew from the study before the experiment was conducted due to a panic situation. The other three subjects withdrew from the study, because they refused to wear VR devices. During follow-up interviews, all participants were assigned numbers that were used in the follow-up interview manuscripts. The numbers range from P1 to P14.

\section{Experimental procedure}

A review of the relevant studies (Anderson et al. 2017; Schutte et al. 2017) found that the proper length of time to wear a VR headset ranged from 6 to $15 \mathrm{~min}$, while the biofeedback instrument generally began collecting data at around $5 \mathrm{~min}$. The MMSE was tested a week ago before the 360-degree garden-video-experience experiment. In addition, the steps to wear and remove the VR headset were not included in the total HRV measurement procedure. The video experience for the experimental procedure was divided into the following (see Fig. 1): (1) attaching the

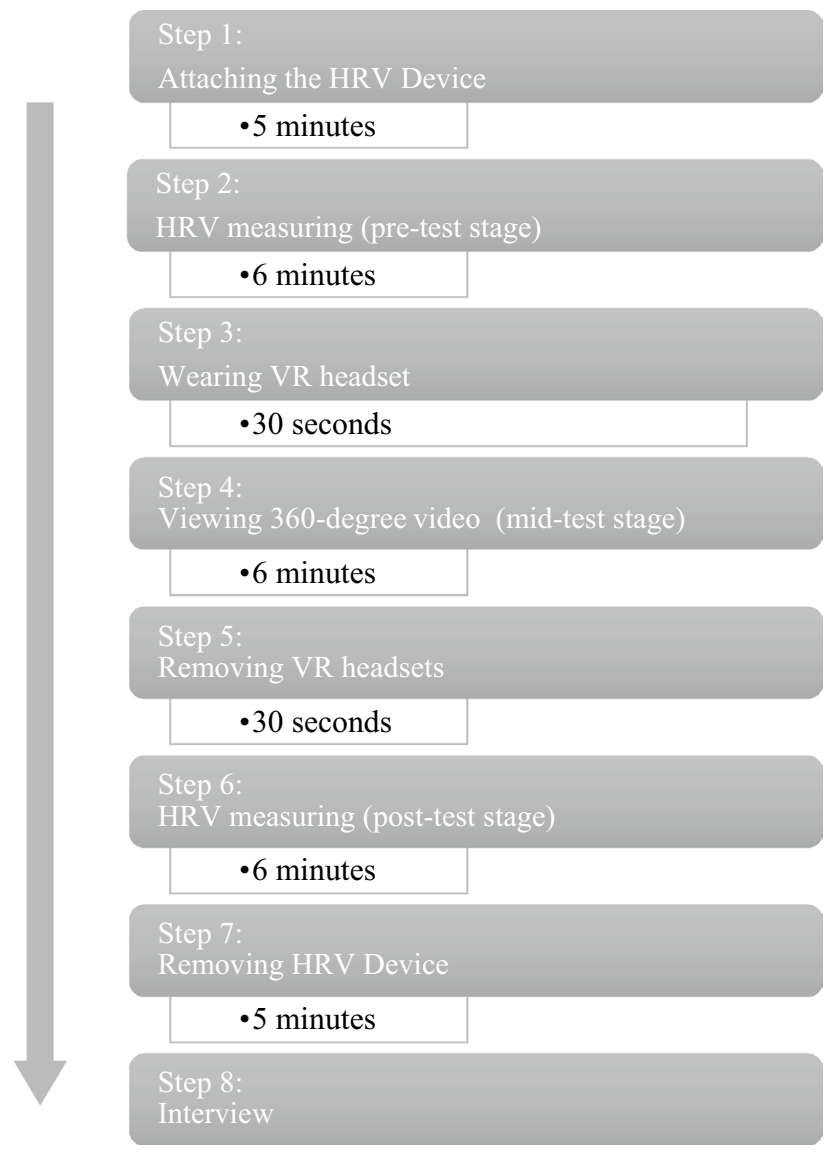

Fig. 1 Experimental procedure in this study
HRV measuring instrument; (2) wearing the VR headset (30 s without measuring HRV) while continuing to measure HRV (6 min); (3) 360-degree garden video experience (6 min); (4) removing the VR headset (30 s without measuring HRV) while continuing to measure HRV (6 min); and (5) interview: receiving an oral response from the participants. The frequency parameters of HRV - LF/HF and SDNNrepresent the balance in the ANS. The Task Force of the European Society of Cardiology and the North American Society of Pacing and Electrophysiology (1996) have stated that a 5 min ECG measurement can estimate the state of an individual's ANS. After referring to a past study (Jiang et al. 2018), the researchers set the pre-test, mid-test, and post-test stages at 6 min to ensure the integrity of the research data.

\section{Research medium, equipment, and testing sites}

The study mainly focused on giving individuals who were not allowed to go outside to experience the outdoors the chance to feel as if they were actually outside through this immersive video experience; this experience was different from watching a $2 \mathrm{D}$ video. This study used a 360-degree camera to shoot the videos. The researchers entered the field site with a GOPRO MAX and tripod. The research fellows took a circular path around the field site and took a 7 min video. The walking speed was in accordance with the behaviors of regular people and the height was set at $160 \mathrm{~cm}$. Efforts were made to prevent videographers from entering the frame, thus making the video appear more authentic (as shown in Fig. 2, left). During the experiment, the researchers selected a location within the nursing home that had the highest degree of feasibility and the lowest degree of interference (including external interference such as sound from a television). A 10/16 $\mathrm{m}$ room with nothing hanging on the walls was selected (as shown in Fig. 2, right).

The location filmed was a vegetable garden located inside the city. In terms of 360-degree video composition, the foreground consisted mainly of a small vegetable garden and gardening facilities, while the middle ground contained a small number of trees and buildings. The background was mostly a clear blue sky (refer to Fig. 3, lower right). Regarding the elements of the vegetable garden, there was a shared community space containing a pavilion, table, and chairs. The garden also contained a few scaffolds, a storage shed for gardening equipment, and watering facilities. The trails were made of a mixture of grass and gravel (refer to Fig. 3, lower left). On both sides of the trail were sponge gourd scaffolds and many neat, rectangular vegetable plots (see Fig. 3, upper right). Each vegetable plot were about three by two meters, and a variety of summer vegetables were being grown, including sponge gourds, sweet potatoes, eggplants, bitter melon, and chayote (see Fig. 3, upper left and right). 


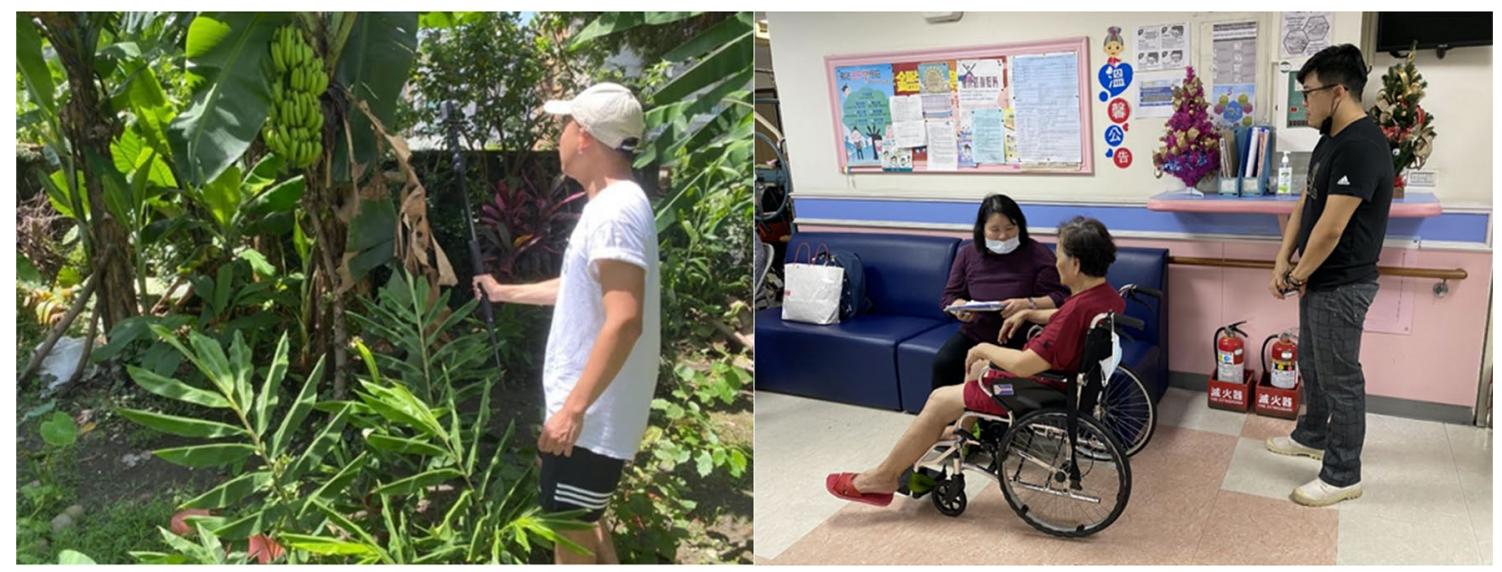

Fig. 2 Filming procedure of the experimental materials and experimental process
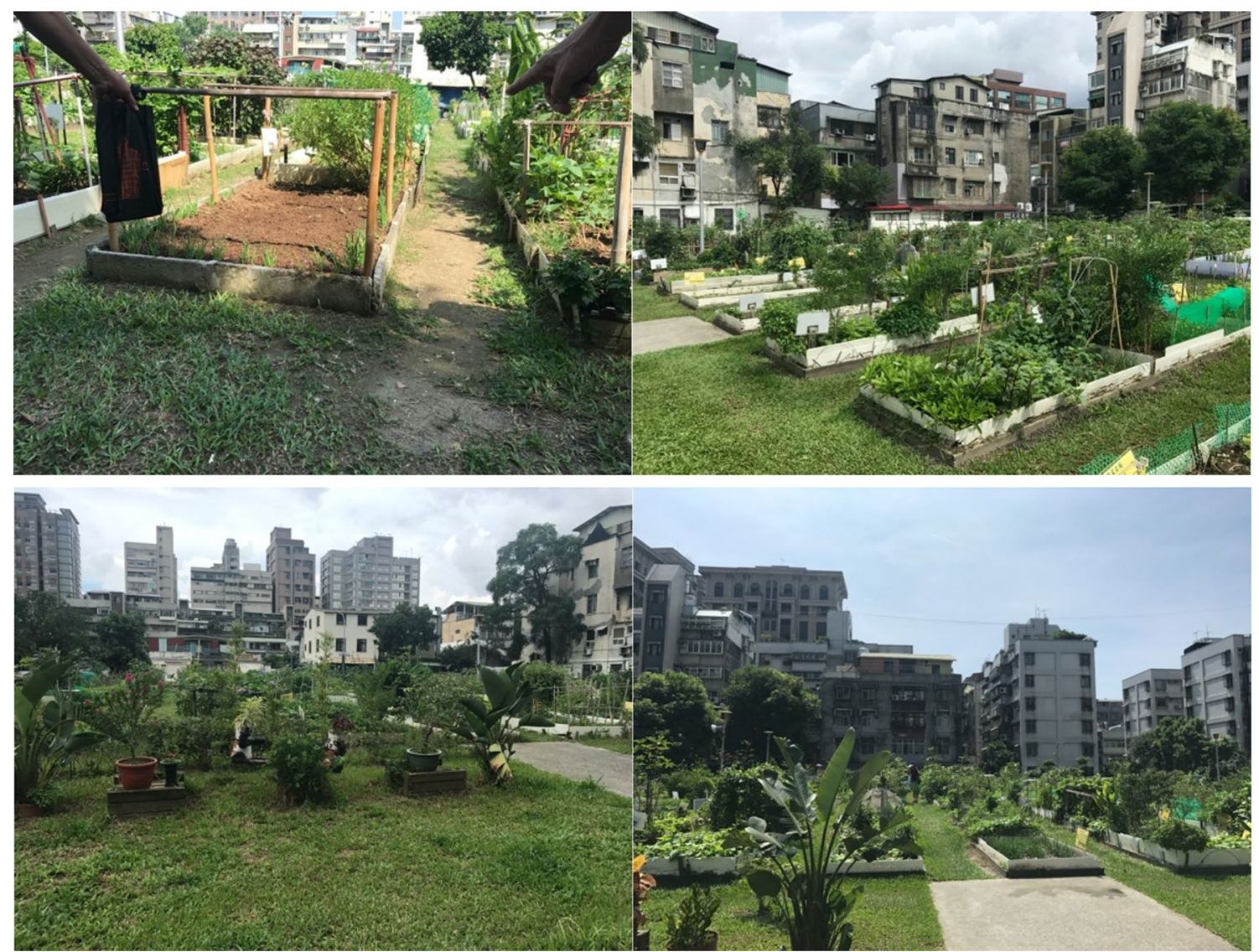

Fig. 3 360-degree video scene diagram

The filming of the garden took place during the summer. This took into account that most elderly people used this space between 6:00 a.m. and 10:00 a.m. or after 4:00 p.m. Therefore, the researchers chose to film the garden during these time slots and did not film when the sun was strongest at noon. The researchers focused on visual elements in the vegetable garden, so, during filming, they avoided having people appear in the actual video (see Fig. 3). In addition, to reduce the impact of sound, noises such as the sound of the videographer or the neighbors were not recorded, while sounds that appeared in the garden, such as insect noises or frog croaks, were preserved. The VR headset chosen for the 
experiment was the Oculus Quest; this device enabled the participants to fully immerse themselves in a high-quality VR. The temperature of the vegetable garden was more than $30{ }^{\circ} \mathrm{C}$, since the filming took place in summer. However, the natural wind and shade made the climate feel much more comfortable. The nursing home experiment site was located indoors and was unable to fully simulate the weather conditions in the outdoor environment. Therefore, the temperature of the room in which the participants viewed the video was kept at $26^{\circ} \mathrm{C}$ to reduce any discomfort caused by overheating. Furthermore, if participants were standing in a real garden, they would be able to smell soil, fertilizer, etc. However, these conditions could not have been replicated in the study, becoming one of its limitations.

\section{Data collection and analysis method}

The study used a BeneGear physiological data sensor, a Bluetooth receiver, and relevant software that had been installed on a laptop to measure HRV. The real-time heart rate and location (LBS) of the wearer could be displayed, allowing researchers to monitor the HRV of the subjects. HRV is related to physiological health and is a balance between the SNS and PNS. Referring to previous literature (Alvarsson et al. 2010; de Kort et al. 2006; Lin et al. 2011), the researchers first measured the subjects' heart rates and then determined the changes in heart rate after implementing the video intervention program. The HRV sensor was attached to the top left quadrant of the subjects' chests. Then, their ECG signals were recorded and converted to LF/HF and SDNN. Next, the laptop received the data using a Bluetooth receiver. The aforementioned data points were continuously measured throughout the experiment to analyze the ANS and HRV. Throughout the process, the research team observed the reactions and expressions of the subjects. Upon completion of the experiment, if the subjects were willing to talk about their experiences, the researchers would conduct an interview.

HRV data was collected and then translated into numerical values, where the data was entered into Microsoft Excel for preliminary organization. Finally, all data were compiled into SPSS 26. Data were collected and translated every $30 \mathrm{~s}$, taking a combined total of $18 \mathrm{~min}$ to collect a subject's full data. The data corresponded to the three different stages: pre-test, mid-test, and post-test. SPSS 26 software was used for statistical analysis. Analysis methods included descriptive statistics and nonparametric statistics. The Wilcoxon signed rank test was used to compare the difference in HRV before, during and after video viewing with VR headsets. The significance value was set at 0.05 . As for the interview portion, the interview content was converted into a manuscript and then summarized.

\section{Results}

This study investigated 14 subjects who successfully completed the experimental procedure and had their LF/HF and SDNN data collected. According to relative studies, the current study in Taiwan (Yang et al. 2018) sets the normal value of the LF/HF ratio between 0.5 and 2.0, where higher than or less than indicates hyperactivity or depression, respectively. The normal value of the SDNN value was set at greater than or equal to 30 . The results are as follows:

\section{Change in HRV and heart rate value in three stages}

Table 1 and Figs. 4-6 show how the values changed before, during, and after viewing the vegetable garden video. In terms of heart rate, there was a continuous downward trend throughout the experiment. Upon completion of the experiment, the average heart rate per minute (79.82) was slightly lower than that in the pre-test stage (80.29) (see Fig. 4). However, the decrease was not significant. The LF value (172.96) showed the highest percentage of increase in the mid-test stage (during video viewing), while the HF value (106.9) exhibited higher values in the post-test stage (see Fig. 5). HF value referred to the activity of PNS. These data changes revealed that subjects might have begun to feel calmer after the experiment. The LF/HF ratio (3.2) in the post-test stage was lower than the pre-test stage ratios (3.66) as it gradually approached the standard range (0.5-2). When comparing the rising ratio in the mid-test and posttest stages (see Fig. 5), the researchers found that the LF value of the participants was higher than their HF during all stages of the experiment. The rising range of the LF/HF ratio increased, showing that the participants felt excited in the mid-test stage. SDNN values were the best during the

Table $1 \mathrm{HRV}$ and heart rate mean and rising ratio in three stages

\begin{tabular}{|c|c|c|c|c|c|c|c|c|c|c|}
\hline & \multicolumn{2}{|l|}{$\mathrm{LF}$} & \multicolumn{2}{|l|}{$\mathrm{HF}$} & \multicolumn{2}{|c|}{$\mathrm{LF} / \mathrm{HF}$} & \multicolumn{2}{|c|}{ SDNN } & \multicolumn{2}{|c|}{ Heart rate } \\
\hline & Mean & Rising ratio & Mean & Rising ratio & Mean & Rising ratio & Mean & Rising ratio & Mean & Rising ratio \\
\hline Pre-test stage & 122.56 & - & 63.14 & - & 3.66 & - & 21.8 & - & 80.29 & - \\
\hline Mid-test stage & 172.96 & 0.411 & 85.5 & 0.354 & 3.96 & 0.082 & 25.57 & 0.173 & 80.19 & -0.001 \\
\hline Post-test stage & 166.32 & 0.253 & 106.9 & 0.512 & 3.2 & -0.116 & 27.3 & 0.215 & 79.82 & -0.006 \\
\hline
\end{tabular}


Fig. 4 Rising ratio in mid-test and post-test stage (color figure online)
Fig. 5 SDNN and heart rate value variation in three stage (color figure online)
$\mathrm{SDNN}(\mathrm{ms})$

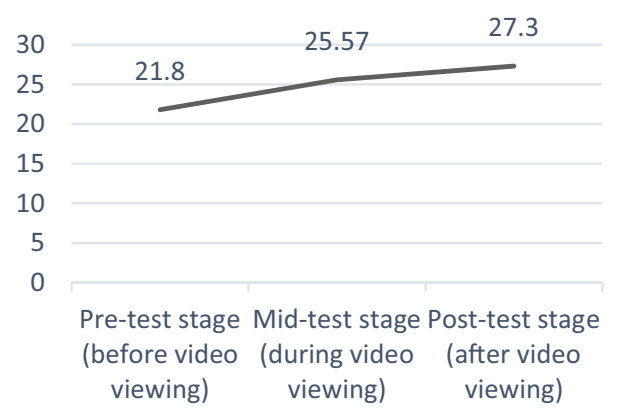

$\longrightarrow \mathrm{LF}(\mathrm{ms} 2) \longrightarrow \mathrm{HF}(\mathrm{ms} 2)$

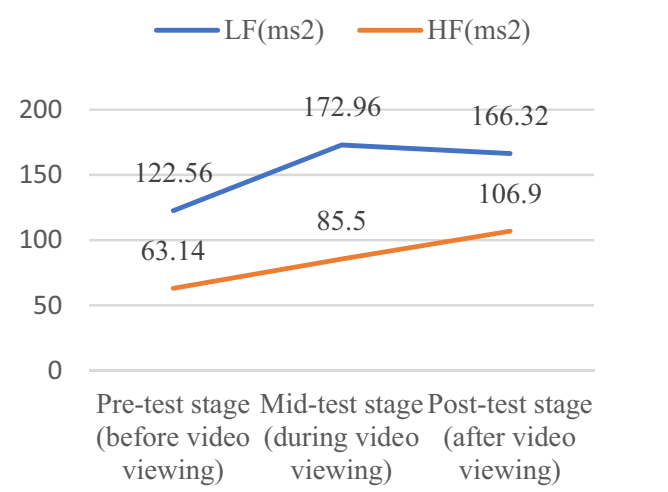

Heart Rate(bpm)

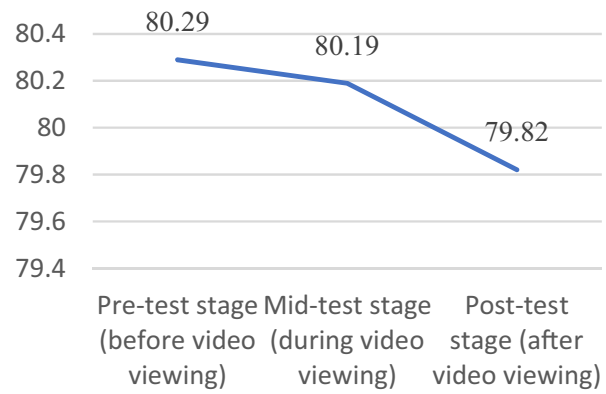

Fig. $6 \mathrm{LH}, \mathrm{HF}$ and LH/HF value variation in three stage (color figure online)

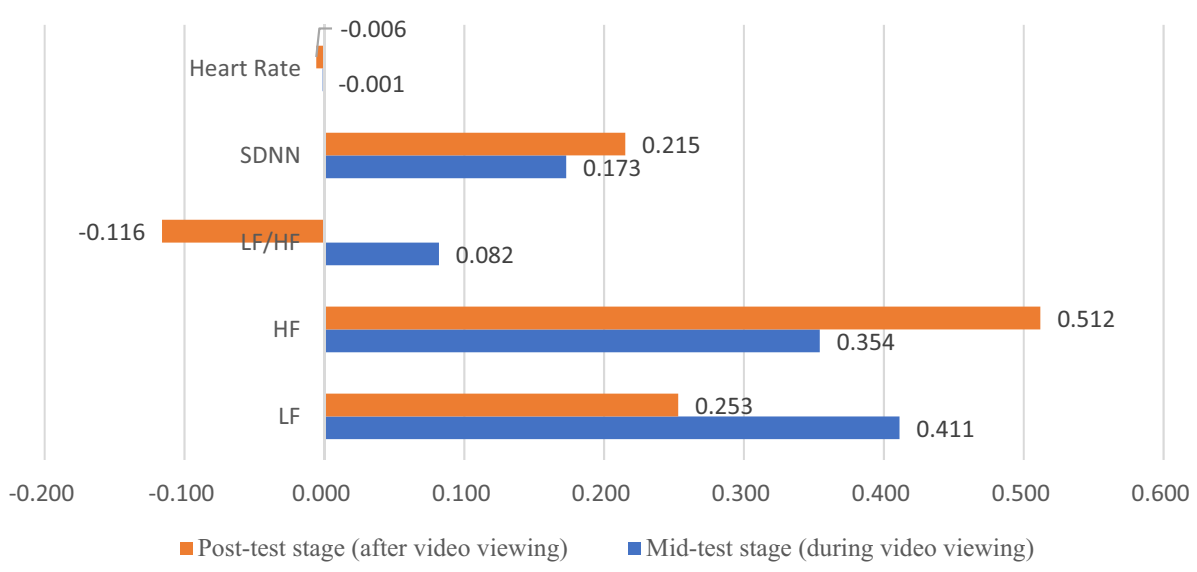

post-test stage, with the participants' heart rates being the fastest during the pre-test stage. Furthermore, the values of the $\mathrm{LF} / \mathrm{HF}$ ratio started to fall in the post-test stage, revealing that the subjects' emotions changed from a state of arousal in the pre-test stage (3.96) to a calmer state (see Fig. 6).

\section{The number of participants changed in the standard HRV range}

As to the number change of participants whose LH/HF ratios were in the standard range (refer to Table 2), three participants were lower than the standard range in LF/HF ratios in the pre-test stage, which represented a dispirited situation.
Eight subjects were above the standard range, which showed a hyper-affectivity situation, and three were in the standard range. During the mid-test stage (during video viewing), two participants were lower than the standard range, seven participants were above, and five participants were in the standard range. The results showed that subjects whose LF/ HF ratios fell outside the standard range decreased after the experiment, and the number of subjects whose $\mathrm{LF} / \mathrm{HF}$ ratios returned to the standard range increased.

In the mid-test stage (during video-viewing), seven LF/ HF ratio scores of the participants increased, while others decreased. To understand the difference of range change between the increase and decrease groups, we divided the 
Table $2 \mathrm{LF} / \mathrm{HF}$ ratio value during the stage of pre-test and mid-test

\begin{tabular}{lllll}
\hline Sample & Pre-test stage & Mid-experiment & Variation & Autonomic nerve situation \\
\hline 1 & $0.43^{*}$ & 0.61 & Increased & Reach to normal range \\
2 & $0.39^{*}$ & $0.34^{*}$ & Decreased & Dispirited \\
3 & $3.77^{\#}$ & $5^{\#}$ & Increased & Hyper-affectivity \\
4 & 0.55 & $2.86^{\#}$ & Increased & Hyper-affectivity \\
5 & $0.42^{*}$ & $0.17^{*}$ & Decreased & Dispirited \\
6 & $3.14^{\#}$ & $4.20^{\#}$ & Increased & Hyper-affectivity \\
7 & 1.93 & 1.38 & Decreased & Steady in normal range \\
8 & $2.02^{\#}$ & 1.99 & Decreased & Steady in normal range \\
9 & $5.72^{\#}$ & $8.42^{\#}$ & Increased & Hyper-affectivity \\
10 & $17.20^{\#}$ & $21.49^{\#}$ & Increased & Hyper-affectivity \\
11 & $3.41^{\#}$ & 1.05 & Decreased & Reduce to normal range \\
12 & $5.29^{\#}$ & $3.34^{\#}$ & Decreased & Hyper-affectivity \\
13 & $4.99^{\#}$ & $4.66^{\#}$ & Decreased & Hyper-affectivity \\
14 & 0.91 & 1.83 & Increased & Steady in normal range \\
\hline
\end{tabular}

\#Above normal range (0.5-2)

*Lower than normal range $(0.5-2)$ participants into two groups-the increase group (seven participants) and the decrease group (seven participants) and then used an independent samples $t$ test to compare the change ranges of these two groups. The results showed that the increase rate of the increase group was significantly higher than that of the decrease group (increase group mean $=1.00 ;$ decrease group mean $=-0.30 ; t=2.379$; $p=0.035$ ).

In terms of SDNN values (refer to Table 3), the SDNN values of the participants were generally low in the pretest stage (before video viewing); 12 participants recorded low SDNN values, while two participants were higher than standard SDNN levels. In the mid-test stage (during video viewing), the SDNN values of 13 participants increased.
Only one participant's SDNN value decreased. The number of participants whose SDNN values were higher than the standard level increased to six after the mid-test stage. The number of participants in the standard level increased by four after implementing the intervention program. The number of participants whose values improved but did not reach a normal level was six. Overall, more than $90 \%$ of the participants had higher SDNN values in the mid-test stage (during video viewing), while four subjects obtained standard SDNN values after the mid-test stage. The results showed that viewing a 360-degree garden video with VR headsets could increase the number of participants with the standard SDNN value.
Table 3 SDNN value during the stage of pre-test and mid-test

\begin{tabular}{lllll}
\hline Sample & Pre-test stage & Mid-experiment & Variation & SDNN situation \\
\hline 1 & $13.57^{\wedge}$ & $7.48^{\wedge}$ & Decreased & Decline \\
2 & $6.97^{\wedge}$ & $8.71^{\wedge}$ & Increased & Active, but did not reach standard value \\
3 & $18.55^{\wedge}$ & $22.47^{\wedge}$ & Increased & Active, but did not reach standard value \\
4 & $3.67^{\wedge}$ & $7^{\wedge}$ & Increased & Active, but did not reach standard value \\
5 & $29.47^{\wedge}$ & 38.84 & Increased & Reached to standard value \\
6 & $10.20^{\wedge}$ & $13.73^{\wedge}$ & Increased & Active, but did not reach standard value \\
7 & $12.52^{\wedge}$ & $22.46^{\wedge}$ & Increased & Active, but did not reach standard value \\
8 & $18.75^{\wedge}$ & 34.68 & Increased & Reached to standard value \\
9 & $20.56^{\wedge}$ & 34.37 & Increased & Reached to standard value \\
10 & $28.38^{\wedge}$ & 32.95 & Increased & Reached to standard value \\
11 & $19.65^{\wedge}$ & 23.04 & Increased & Active, but did not reach standard value \\
12 & 31.41 & 38.72 & Increased & Active \\
13 & 42.98 & 60.71 & Increased & Active \\
14 & $16.03^{\wedge}$ & $26.89^{\wedge}$ & Increased & Active, but did not reach standard value \\
\hline
\end{tabular}

${ }^{\wedge}$ Lower than standard value (30) 


\section{The paired-samples $t$ test of LF/HF and SDNN among pre-, mid-, and post-test stages}

To understand whether viewing a 360-degree video with VR headsets could significantly affect SDNN value and LF/ $\mathrm{HF}$ ratio, we used the Wilcoxon signed rank test to compare whether there were statistical differences in HRV during the pre-test stage, mid-test stage, and post-test stage. The results showed that there were no significant differences in LF/HF, but there was a significant difference in the SDNN values (refer to Table 4). SDNN values during and after the experiment were higher than SDNN values before the experiment.

\section{Content of the interview after immersive garden experience}

In this study, the research fellows invited the participants to express their thoughts and feelings after the experiment. After summarizing the interviews, it was found that the videos the participants watched reminded them of their past and allowed them to relive happy moments. The result of this study found that viewing a 360-degree video with VR headsets provided an alternative outdoor experience for those who could not leave the nursing home due to COVID-19. This intervention program allowed participants to feel as if they were actually experiencing nature.

I can't leave this place, so watching this video makes me feel as if I'm outside. I feel so at ease watching this video, and I'm very happy that I was able to see it. $-P 6$

After watching the video, I felt pretty happy. I want to go outside and enjoy myself. It was very relaxing. -P8

After the video viewing, the participants also brought up their past memories of spending time together with their family.

I used to have a small vegetable garden when I lived in the countryside.... I planted many kinds of vegetables like sweet potato leaves, lima beans, green beans, potatoes, water spinach.... This video of the small garden really brings me back to those days I used to spend back in my garden. Ah... what fond memories. - P4
The garden was everywhere in the video; it really brings me back... I remember when my family and I would go pick vegetables, and then I would cook them. - P10

Watching the video really brought back memories for me. I have also planted sweet potatoes leaves and sponge gourds before. It also looked like there were peanut plants. When I was living in the South, I used to plant peanut plants. It brings me great joy to see these things from my past. $-P 12$.

\section{Conclusions and discussion}

\section{Conclusion}

In this study, we developed a 360-degree vegetable garden video using 360-degree photography equipment and provided an immersive experience with VR headsets, allowing participants to virtually come into contact with vegetable garden scenes. We also used multiple methods to investigate psychological and physiological responses on health outcomes. HRV data was collected using a biofeedback instrument and interviews were conducted after the experiment. These results demonstrated that the 360-degree vegetablegarden video could have short-term cardiovascular benefits and might help release stress. The change in HRV parameters showed that this video had a positive effect on the increase in SDNN and HF values. After patients viewed the 360-degree video, the changes in $\mathrm{LF} / \mathrm{HF}$ ratio values tended to fall between 0.5 and 2 , helping hold firm the participants' LF/HF ratio ranges. Most of the participants' LF/HF ratios either improved or remained at a normal level. In the LF/HF ratio measurement result, the experiment increased the LF/ HF ratio of half the participants, and the range rate of the increase group was significantly higher than the decrease group. Thus, the 360-degree video might be better able to arouse the participants' emotions and interests than to help calm and relax their moods.

Throughout this experiment, nearly $92 \%$ of the participants showed an increase in their SDNN values. While comparing the HRV values of all three stages, the researchers found through the Wilcoxon signed-rank test that the SDNN values in the mid-test stage and post-test stage were
Table 4 SDNN value in three stages of the Wilcoxon signedrank test

\begin{tabular}{lllllll}
\hline Verified content & Stage & $M$ & S.D & $N$ & $Z$ & $P$ \\
\hline Pre-test stage & Pre-test stage (6 min) & 21.80 & 10.990 & 14 & 2.229 & $0.026^{*}$ \\
Mid-test stage & Mid-test stage (6 min) & 25.56 & 14.669 & 14 & & \\
Mid-test stage & Mid-test stage (6 min) & 25.56 & 14.669 & 14 & 2.229 & $0.026^{*}$ \\
Post-test stage & Post-test stage (6 min) & 27.47 & 17.235 & 14 & & \\
\hline
\end{tabular}

${ }^{*} p<0.05$ 
significantly higher than during the pre-test stage. This study found that after VR headsets were removed, SDNN and HF values remained in an increased state for at least $6 \mathrm{~min}$ rather than immediately dropping. The results also hinted that the health benefit of viewing the 360-degree garden video could still last for a certain period. Based on the results of our interviews, we found that our video could awaken the interest of the participants. The participants had positive psychological responses to the vegetable crops in the vegetable garden, which reminded these participants of wonderful memories of their lives, such as childhood in the country. Overall, our findings are as follows: (1) the effect of viewing a 360-degree garden video on increasing the SDNN values of patients was greater than the other HRV parameters, which demonstrated that viewing a 360-degree garden video had a more direct effort on SDNN and (2) the 360-degree video could arouse participants' positive emotions and remind them of wonderful memories from their lives.

\section{Discussion}

Since the outbreak of COVID-19, the mobility of elderly residents living in nursing homes has limited access to nature more than the daily life of their pasts. The restriction of going out for nature exposure and exercise increases the chances of cardiovascular diseases for elderly residents living in nursing homes. Relative studies (Browning et al. 2020; Jerdan et al. 2018; Maples-Keller et al. 2017; Valtchanov and Ellard 2015; White et al. 2018) have shown that panoramic videos were able to improve an individual's emotional state. Li et al. (2021) proved that VR experiences can help increase exercise pleasure, buffer heart rate, and improve cardiac autonomic recovery for normal males. Yu et al. (2020) emphasized the health benefits of VR on middle-aged and elderly adults with normal mental conditions.

We selected participants with mild-to-moderate cognitive impairment living in nursing homes. The participants were restricted from going outside by nursing home regulations due to the COVID-19 outbreak in Taiwan. Most of the participants were in negative and unstable emotional states. Kop et al. (2010) emphasized that negative emotions might increase the risk of cardiovascular diseases. As a result, providing an alternative outdoor experience for those who could not leave the nursing home could help them stay healthy (Yu et al. 2020). The results of our experiment showed that SDNN values increased while viewing the 360 -degree video. Most of the participants expressed that they were satisfied with this experiment. This means that VR experiences can increase the positive emotions of dementia patients, allowing them to be calmer and more stable.

In this study, we developed a way for people who are restricted from the outside to contact the landscape they preferred, and we also showed that viewing a 360-degree vegetable garden video indoors was of great benefit to the residents of the LTC facility who were restricted from going out. As a result, we believe viewing panoramic videos with VR headsets might benefit people who are under home isolation or quarantine.

The benefits of viewing a panoramic garden video with VR headsets have been proven in our study. Manera et al. (2016) indicated that participants with dementia were highly satisfied and interested in their VR experiment, and reported high feelings of security and low discomfort, anxiety, and fatigue. Although some participants were unable to answer the questions clearly in our experiment, their facial expressions and behaviors still revealed their pleasure and willingness to view the VR video. A relative study (Man et al. 2012) indicated that VR experience could result in greater improvement in objective memory performance. Our study also demonstrated that providing such videos with VR headsets could help participants remember their happy memories, which might help improve cognitive performance.

In the current study, the vegetable crops in the panoramic video were found to be important in improving the positive responses of the participants. Kemperman and Timmermans (2014) pointed out that the elderly prefer vegetable gardens, where they can garden and talk to other people every day. We chose the vegetable garden in Taipei City as the resource for panoramic video shooting and confirmed that all elements of the garden except humans were filmed as much as possible. The interview results showed that after viewing the video, the participants had positive responses to vegetables, such as sponge gourds on tent-shaped trellises, sweet potato leaves, and peanuts, which they used to grow in the past. Relative studies (Souter-Brown et al. 2021; Uwajeh et al. 2019) emphasized the health outcomes of contact with garden crops in a therapeutic garden. Wood et al. (2015) highlighted that memories of spaces can impinge on one's present therapeutic experience. Lin et al. (2018) believed that city gardens can corporeally interact with nature elements to develop greater fascination with nature. We proved that vegetables crops can increase positive health outcomes, help participants recall memories, and attract elderly people living in LCT facilities, just like waterscapes mentioned in a relative study (Xie et al. 2021). Thus, the element of garden crops should be taken into account when designing realistic or virtual therapeutic gardens to enhance the quality of life and well-being of the elderly.

\section{Future consideration}

In this research, HRV data and data from in-depth interviews conducted after the experiment were collected. Only five participants were able to successfully be interviewed, because the others were either suffering from cognitive 
impairment, were unable to undergo the interview, or could not fully express themselves. In view of this, other instruments could be introduced in the future, or further interview analysis methods could be conducted to better understand the main elements that the participants pay attention to during the video experiment. This will allow follow-up studies to design or select more suitable landscapes for patients with dementia.

In our experiment, the video elicited a positive emotional reaction. These positive responses could be attributed to the novelty of the VR experience rather than the interest of the participants in the landscape. In fact, during the experiment, a few participants felt that they were not interested in the garden landscape and had a negative response or gave up halfway. Hodge et al. (2018) highlighted the importance of the participation of dementia patients in the VR environment design process. Therefore, if research fellows can have a better understanding of the participants' preferences or let them choose their preferred VR videos, then participants might have more interest in the video-viewing experiment. Furthermore, this would also increase the interactions and social opportunities of participants.

Supplementary Information The online version contains supplementary material available at https://doi.org/10.1007/s11355-021-00480-9.

Funding This study was supported by the Ministry of Science and Technology of Taiwan (108-2410-H-030-068-SSS).

\section{Declarations}

Conflict of interest The authors declare that there are no conflicts of interest.

\section{References}

Alvarsson JJ, Wiens S, Nilsson ME (2010) Stress recovery during exposure to nature sound and environmental noise. Int J Environ Res Public Health 7:1036-1046. https://doi.org/10.3390/ijerp h7031036

Anderson AP, Mayer MD, Fellows AM, Cowan DR, Hegel MT, Buckey JC (2017) Relaxation with immersive natural scenes presented using virtual reality. Aerosp Med Hum Perform 88:520-526. https://doi.org/10.3357/amhp.4747.2017

Bilchick KC, Fetics B, Djoukeng R, Fisher SG, Fletcher RD, Singh SN et al (2002) Prognostic value of heart rate variability in chronic congestive heart failure (veterans affairs' survival trial of antiarrhythmic therapy in congestive heart failure). Am J Cardiol 90:24-28. https://doi.org/10.1016/s0002-9149(02)02380-9

Browning MHEM, Mimnaugh KJ, van Riper CJ, Laurent HK, LaValle SM (2020) Can simulated nature support mental health? Comparing short, single-doses of 360-degree nature videos in virtual reality with the outdoors. Front Psychol. https://doi.org/10.3389/ fpsyg.2019.02667

Catai AM, Pastre CM, Godoy MF, Silva ED, Takahashi ACM, Vanderlei LCM (2020) Heart rate variability: are you using it properly?
Standardisation checklist of procedures. Braz J Phys Ther 24:91102. https://doi.org/10.1016/j.bjpt.2019.02.006

Chiu HY, Chien YN, Lu JF, Kuo CL, Hsu CY, Li HA, Hao CJ, Li LW (2019) The financial challenge of health care and its response in an elderly society. Specific Report of CTCI Foundation 2019-03

Craske MG, Meuret AE, Ritz T, Treanor M, Dour H, Rosenfield D (2019) Positive affect treatment for depression and anxiety: a randomized clinical trial for a core feature of anhedonia. J Consult Clin Psychol 87:457-471. https://doi.org/10.1037/ccp0000396

de Kort YAW, Meijnders AL, Sponselee AAG, Ijsselsteijn WA (2006) What's wrong with virtual trees? Restoring from stress in a mediated environment. J Environ Psychol 26:309-320. https://doi.org/ 10.1016/j.jenvp.2006.09.001

Doniger GM, Beeri MS, Bahar-Fuchs A, Gottlieb A, Tkachov A, Kenan $\mathrm{H}$ et al (2018) Virtual reality-based cognitive-motor training for middle-aged adults at high Alzheimer's disease risk: a randomized controlled trial. Alzheimer's Dementia 4:118-129. https://doi.org/10.1016/j.trci.2018.02.005

Economic and Social Commission for Asia and the Pacific (2016) Long term care for older persons in China. https://www.unescap.org/ sites/default/files/Long\%20Term\%20Care\%20for\%20older\%20per sons\%20in\%20China.pdf. Accessed 1 Apr 2021

Folstein MF, Folstein SE, McHugh PR (1975) "Mini-mental state": a practical method for grading the cognitive state of patients for the clinician. J Psychiatric Res 12:189-198. https://doi.org/10.1016/ 0022-3956(75)90026-6

Harris-Kojetin L, Sengupta M, Lendon JP, Rome V, Valverde R, Caffrey C (2019) Long-term care providers and services users in the United States, 2015-2016. National Center for Health Statistics. Vital and Health Statistics 3. https://www.cdc.gov/nchs/data/ series/sr_03/sr03_43-508.pdf. Accessed 3 Apr 2021

Health Promotion Administration (2017) National health insurance medical statistics annual report. https://dep.mohw.gov.tw/DOS/ np-4267-113.html. Accessed 6 Apr 2021

Health Promotion Administration (2018) 2016 annual report. https:// www.hpa.gov.tw/Pages/Detail.aspx .nodeid $=1249 \&$ pid $=7084$. Accessed 5 Apr 2021

Hoben M, Heninger A, Holroyd-Leduc J, Knopp-Sihota J, Estabrooks C, Goodarzi Z (2019) Depressive symptoms in long term care facilities in Western Canada: a cross sectional study. BMC Geriatr 19:335. https://doi.org/10.1186/s12877-019-1298-5

Hodge J, Balaam M, Hastings S, Morrissey K (2018) Exploring the design of tailored virtual reality experiences for people with dementia. In: Proceedings of the 2018 CHI Conference on Human Factors in Computing Systems (Paper 514): Association for Computing Machinery

Huang HT, Chuang YH, Hsueh YH, Lin PC, Lee BO, Chen CH (2014) Depression in older residents with stroke living in long-term care facilities. J of Nurs Res 22:111-118. https://doi.org/10.1097/jnr. 0000000000000028

Jerdan SW, Grindle M, van Woerden HC, Kamel Boulos MN (2018) Head-mounted virtual reality and mental health: critical review of current research. JMIR Serious Games 6:e14. https://doi.org/ 10.2196/games.9226

Jiang B, Schmillen R, Sullivan WC (2018) How to waste a break: using portable electronic devices substantially counteracts attention enhancement effects of green spaces. Environ Behav 51:11331160. https://doi.org/10.1177/0013916518788603

Jongenelis K, Pot AM, Eisses AMH, Beekman ATF, Kluiter H, Ribbe MW (2004) Prevalence and risk indicators of depression in elderly nursing home patients: the AGED study. J Affect Disord 83:135142. https://doi.org/10.1016/j.jad.2004.06.001

Kemperman A, Timmermans H (2014) Green spaces in the direct living environment and social contacts of the aging population. Landsc Urban Plan 129:44-54. https://doi.org/10.1016/j.landu rbplan.2014.05.003 
Kim A, Darakjian N, Finley JM (2017) Walking in fully immersive virtual environments: an evaluation of potential adverse effects in older adults and individuals with Parkinson's disease. J NeuroEng Rehabil 14:16. https://doi.org/10.1186/s12984-017-0225-2

Kleiger RE, Miller JP, Bigger JT Jr, Moss AJ (1987) Decreased heart rate variability and its association with increased mortality after acute myocardial infarction. Am J Cardiol 59:256-262. https:// doi.org/10.1016/0002-9149(87)90795-8

Kop WJ, Stein PK, Tracy RP, Barzilay JI, Schulz R, Gottdiener JS (2010) Autonomic nervous system dysfunction and inflammation contribute to the increased cardiovascular mortality risk associated with depression. Psychosom Med 72:626-635. https://doi. org/10.1097/PSY.0b013e3181eadd2b

Kurita A, Takase B, Kodani E, Iwahara S, Kusama Y, Atarashi H (2013) Prognostic value of heart rate variability in comparison with annual health examinations in very elderly subjects. J Nippon Med Sch 80:420-425. https://doi.org/10.1272/jnms.80.420

La Rovere MT, Bigger JT Jr, Marcus FI, Mortara A, Schwartz PJ (1998) Baroreflex sensitivity and heart-rate variability in prediction of total cardiac mortality after myocardial infarction. ATRAMI (autonomic tone and reflexes after myocardial infarction) investigators. Lancet 351:478-484. https://doi.org/10.1016/s01406736(97)11144-8

Lee DT, Woo J, Mackenzie AE (2002) A review of older people's experiences with residential care placement. J Adv Nurs 37:19-27. https://doi.org/10.1046/j.1365-2648.2002.02060.x

Lee VSP, Simpson J, Froggatt K (2013) A narrative exploration of older people's transitions into residential care. Aging Ment Health 17:48-56. https://doi.org/10.1080/13607863.2012.715139

Li H, Zhang X, Bi S, Liu H, Cao Y, Guodong Z (2021) Green exercise: can nature video benefit isometric exercise? Int J Environ Res Public Health 18:5554. https://doi.org/10.3390/ijerph18115554

Lin CC, Chuang CM, Chao SY, Lee CK (2007) A study on functional assessment in long-term care facility. Hungkuang Academic Rev 52:67-80

Lin HP, Lin HY, Lin WL, Huang ACW (2011) Effects of stress, depression, and their interaction on heart rate, skin conductance, finger temperature, and respiratory rate: sympathetic-parasympathetic hypothesis of stress and depression. J Clin Psychol 67:1080-1091. https://doi.org/10.1002/jclp.20833

Lin BB, Egerer MH, Ossola A (2018) Urban gardens as a space to engender biophilia: evidence and ways forward. Frontiers Built Environ. https://doi.org/10.3389/fbuil.2018.00079

Liszio S, Graf L, Masuch M (2018) The relaxing effect of virtual nature: immersive technology provides relief in acute stress situations. Annu Rev CyberTherap Telemed 16:87-93

Man DWK, Chung JCC, Lee GYY (2012) Evaluation of a virtual reality-based memory training programme for Hong Kong Chinese older adults with questionable dementia: a pilot study. Int J Geriatric Psychiatry 27:513-520. https://doi.org/10.1002/gps.2746

Manera V, Chapoulie E, Bourgeois J, Guerchouche R, David R, Ondrej $\mathrm{J}$ et al (2016) A feasibility study with image-based rendered virtual reality in patients with mild cognitive impairment and dementia. PLoS One 11:e0151487. https://doi.org/10.1371/journal.pone. 0151487

Maples-Keller JL, Bunnell BE, Kim SJ, Rothbaum BO (2017) The use of virtual reality technology in the treatment of anxiety and other psychiatric disorders. Harv Rev Psychiatry 25:103-113. https:// doi.org/10.1097/hrp.0000000000000138
Mattila O, Korhonen A, Pöyry E, Hauru K, Holopainen J, Parvinen P (2020) Restoration in a virtual reality forest environment. Comput Hum Behav 107:106295. https://doi.org/10.1016/j.chb.2020. 106295

Palanica A, Lyons A, Cooper M, Lee A, Fossat Y (2019) A comparison of nature and urban environments on creative thinking across different levels of reality. J Environ Psychol 63:44-51. https://doi. org/10.1016/j.jenvp.2019.04.006

Schermer M (2014) Transnational at home: intercultural gardens and the social sustainability of cities in Innsbruck. Austria Hábitat y Sociedad. https://doi.org/10.12795/HabitatySociedad.2013.i7.04

Schutte NS, Bhullar N, Stilinović EJ, Richardson K (2017) The impact of virtual environments on restorativeness and affect. Ecopsychology 9:1-7. https://doi.org/10.1089/eco.2016.0042

Souter-Brown G, Hinckson E, Duncan S (2021) Effects of a sensory garden on workplace wellbeing: a randomised control trial. Landsc Urban Plan 207:103997. https://doi.org/10.1016/j.landu rbplan.2020.103997

Task Force of the European Society of Cardiology and the North American Society of Pacing and Electrophysiology (1996) Heart rate variability: standards of measurement, physiological interpretation, and clinical use. Eur Heart J 17:354-381. https://doi.org/10. 1093/oxfordjournals.eurheartj.a014868

Uwajeh PC, Iyendo TO, Polay M (2019) Therapeutic gardens as a design approach for optimising the healing environment of patients with Alzheimer's disease and other dementias: a narrative review. Explore 15:352-362. https://doi.org/10.1016/j.explo re.2019.05.002

Valtchanov D, Ellard CG (2015) Cognitive and affective responses to natural scenes: effects of low level visual properties on preference, cognitive load and eye-movements. J Environ Psychol 43:184195. https://doi.org/10.1016/j.jenvp.2015.07.001

Victor CR (2012) Loneliness in care homes: a neglected area of research? Aging Health 8:637-646. https://doi.org/10.2217/ahe. 12.65

White MP, Yeo NL, Vassiljev P, Lundstedt R, Wallergård M, Albin M, Lõhmus M (2018) A prescription for "nature"-the potential of using virtual nature in therapeutics. Neuropsychiatric Dis Treat 14:3001-3013. https://doi.org/10.2147/ndt.s179038

Wood VJ, Gesler W, Curtis SE, Spencer IH, Close HJ, Mason J, Reilly JG (2015) 'Therapeutic landscapes' and the importance of nostalgia, solastalgia, salvage and abandonment for psychiatric hospital design. Health Place 33:83-89. https://doi.org/10.1016/j.healt hplace.2015.02.010

Xie Q, Lee C, Lu Z, Yuan X (2021) Interactions with artificial water features: a scoping review of health-related outcomes. Landsc Urban Plan 215:104191. https://doi.org/10.1016/j.landurbplan. 2021.104191

Yang CH, Chen HY, Chen JL, Yang SH (2018) Therapeutic efficacy of traditional Chinese medicine on quality of life and heart rate variability among patients in long-term care system in Taiwan. J Chin Med 29:42-59. https://doi.org/10.3966/241139642018062901004

Yu CP, Lee HY, Lu WH, Huang YC, Browning MHEM (2020) Restorative effects of virtual natural settings on middle-aged and elderly adults. Urb For Urb Gr 56:126863. https://doi.org/10.1016/j.ufug. 2020.126863 